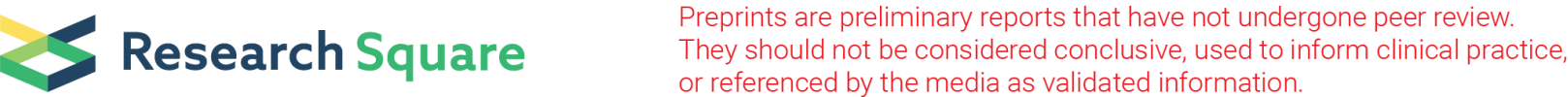

\section{The Efficacy of Casirivimab/Imdevimab in Solid Organ Transplant Recipients with Mild-To-Moderate COVID-19}

\section{Etsuko Tagaya}

Tokyo Women's Medical University

Ken Kikuchi

Tokyo Women's Medical University

\section{Toshihiro Mitsuda}

Tokyo Women's Medical University

\section{Fumiya Ebihara}

Tokyo Women's Medical University

\section{Takumi Maruyama}

Tokyo Women's Medical University

\section{Yukihiro Hamada}

Tokyo Women's Medical University

Toshimi Kimura

Tokyo Women's Medical University

Kohei Unagami

Tokyo Women's Medical University

Taichi Kanzawa

Tokyo Women's Medical University

Haruki Sekiguchi

Tokyo Women's Medical University

\section{Ken Shimamoto}

Tokyo Women's Medical University

\section{Hideki Ishida}

Tokyo Women's Medical University

\section{Hiroto Egawa}

Tokyo Women's Medical University

Junji Tanaka

Tokyo Women's Medical University

Kazunari Tanabe

Tokyo Women's Medical University

Masatoshi Kawana 
Tokyo Women's Medical University

Ken Arimura ( $\sim$ arimuraken@gmail.com )

Tokyo Women's Medical University

\section{Research Article}

Keywords: Casirivimab/imdevimab, SOTR, SARS-CoV-2, COVID-19, neutralizing antibody

Posted Date: March 1st, 2022

DOI: https://doi.org/10.21203/rs.3.rs-1361782/v1

License: (c) (1) This work is licensed under a Creative Commons Attribution 4.0 International License. Read Full License 


\section{Abstract}

Background: Solid organ transplant recipients (SOTRs) are at high risk for severe coronavirus disease 2019 (COVID-19) due to comorbidities and immunosuppression. Moreover, the treatment for COVID-19 is limited. Neutralizing monoclonal antibodies (mAbs) against severe acute respiratory syndrome coronavirus 2 are used for treating mild-to-moderate COVID-19. However, reports comparing the efficacy of COVID-19 treatment with and without neutralizing mAbs in SOTRs are limited. Here, we assessed the efficacy of casirivimab/imdevimab as neutralizing mAbs against mild-to-moderate COVID-19 in SOTRs.

Methods: Forty-one patients hospitalized from September 2020 to September 2021 at Tokyo Women's Medical University Hospital were included. The duration until antifebrile and of hospitalization, requirement of oxygen $\left(\mathrm{O}_{2}\right) \geq 5 \mathrm{~L}$, and neutralizing antibody levels were compared in SOTRs with COVID19 who received and those who did not receive casirivimab/imdevimab therapy.

Results: Casirivimab/imdevimab therapy correlated with less duration until antifebrile and of hospitalization and non-requirement of $\mathrm{O}_{2} \geq 5 \mathrm{~L}$ in SOTRs with COVID-19 [mean days without vs. with casirivimab/imdevimab: 6 vs. $2 ; P=0.0002$, hazard ratio $(\mathrm{HR})=0.3333,95 \%$ confidence interval $(\mathrm{Cl})=$ $0.1763-0.6301$; mean days: 15 vs. $8 ; P<0.0001, \mathrm{HR}=0.5333,95 \% \mathrm{Cl}=0.2878-0.9883 ; P=0.0377, \mathrm{HR}=$ $0.1502,95 \% \mathrm{Cl}=0.02511-0.8980$, respectively]. Further, casirivimab/imdevimab therapy was associated with early antifebrile after adjusting for sex and age $(P=0.013, \mathrm{HR}=0.412,95 \% \mathrm{Cl}=0.205-0.826)$. Although no significant difference was observed in neutralizing antibody levels between patients without and with casirivimab/imdevimab therapy on the day of hospitalization $(P=0.1055)$, including 13 SOTRs who had received vaccination. Neutralizing antibody levels were higher in patients with casirivimab/imdevimab therapy at 3-5 days after hospitalization including such patients in whom the levels tended to stay lower despite vaccination than in those without casirivimab/imdevimab at 7-9 days after hospitalization ( $P<0.0001$, mean antibody without vs. with, 414.9 vs. 40000$)$.

Conclusion: Casirivimab/imdevimab therapy was effective and increased the neutralizing antibody levels in SOTRs with mild-to-moderate COVID-19. Therefore, casirivimab/imdevimab therapy could contribute to preventing the progression of mild-to-moderate COVID-19 in SOTRs.

\section{Background}

Coronavirus disease 2019 (COVID-19) derived from severe acute respiratory syndrome coronavirus 2 (SARS-CoV-2) has spread globally [1]. As of December 31, 2021, the number of 5393,950 deaths cases have been reported in the world [2].

Patients with solid organ transplants, diabetes mellitus, cardiovascular disease, chronic obstructive pulmonary disease, and cancer have a worse prognosis after COVID-19 [3,4]. In particular, solid organ transplant recipients (SOTRs) are at high risk for severe COVID-19 due to comorbidities and immunosuppression [5]. Although the treatment for COVID-19 is limited, various combination therapies are explored, including remdesivir as an anti-viral agent, dexamethasone, tocilizumab, and baricitinib as 
an anti-inflammation agent [6-9]. Furthermore, BNT162b2 and mRNA-1273 have been employed as vaccines to prevent SARS-CoV-2 infection and progression [10,11]. Despite these options, SOTRs exhibit a worse prognosis [12] and less antibody response due to immunosuppression [13] than in patients without SOTRs.

Casirivimab/imdevimab, bamlanivimab/etsevimab, and sotrovimab are neutralizing monoclonal antibodies (mAbs) against the surface spike protein of SARS-CoV-2 and have been used for the treatment of mild-to-moderate COVID-19 [14-16]. Neutralizing mAbs reduce SARS-CoV-2 viral load, hospitalization, and death in patients with diabetes mellitus, cardiovascular disease, various chronic diseases, and immunosuppression [14-16]. However, only letters or brief reports have reported the data for COVID-19 in SOTRs in terms of hospitalization, prognosis, and tolerance with $[5,17]$ or without $[18-22]$ neutralizing mAbs therapy, and the studies on the comparison of therapies with and without neutralizing mAbs for COVID-19 in SOTRs are limited. We hypothesized that neutralizing mAbs therapy would be more effective to treat COVID-19 in SOTRs than that without neutralizing mAbs. In this study, we assessed retrospectively the clinical impact of therapies with and without casirivimab/imdevimab neutralizing mAbs in SOTRs with COVID-19.

\section{Methods}

\section{Ethical considerations}

This retrospective study was conducted using data retrieved from patients' medical records from September 2020 to September 2021 at the Tokyo Women's Medical University Hospital. This study was approved by the institutional review board (date of approval: January 5, 2022; approval number: 2021 0175) at Tokyo Women's Medical University. The board disclosed this study on the institution website. Informed consent was obtained from all patients at the time of hospitalization.

\section{Patients}

All patients were eligible for the therapy with neutralizing mAbs. The included patients were SOTRs with mild-to-moderate COVID-19 and were hospitalized within 1 week after the onset. One group received casirivimab/imdevimab intravenously immediately after hospitalization. The other group were eligible for neutralizing mAbs therapy; however, they were not administered casirivimab/imdevimab because they infected the SARS-CoV-2 before the appearance of the neutralizing mAb. Mild-to-moderate COVID-19 was defined according to the Food and Drug Administration criteria [23]. Briefly, the symptoms and clinical signs of mild-to-moderate of COVID-19 could include fever, cough, sore throat, headache, malaise, muscle pain, nausea, vomiting, diarrhea, loss of taste or smell, respiratory rate $\geq 20$ breaths per min, heart rate $\geq$ 90 beats per min, and saturation of oxygen $\left(\mathrm{SpO}_{2}\right) \geq 94 \%$ on room air at sea level. The discharge was decided when the clinical symptoms disappeared, including body temperature $<37^{\circ} \mathrm{C}$ for $72 \mathrm{~h}$ and $\mathrm{SpO}_{2}$ $\geq 94 \%$ on room air [23].

\section{Polymerase chain reaction}


All SOTRs were tested for SARS-CoV-2 with polymerase chain reaction (PCR) before hospitalization. Even if the patients had tested at another institution, they were tested again. PCR was performed as we previously described [24]. Briefly, specimens were sampled from the nasal cavities of patients with swabs (503CS01-E, BP100; Shin Corporation, Hyogo, Japan). After viral ribonucleic acid was extracted with the QIAamp Viral RNA Mini Kit (QIAGEN, Tokyo, Japan), PCR was conducted as per the manufacturer's instructions using the amplification reagent (BGI Genomics, Shenzhen, China).

\section{Neutralizing antibody levels}

Neutralizing antibody levels in serum were measured using the Alinity SARS-CoV-2 IgG II Quant kit (Abbot, Chicago, IL, USA) in all patients. The effective level of neutralizing antibody as an anti-SARS-CoV-2 threshold was stipulated to be $\geq 4160 \mathrm{AU} / \mathrm{mL}$, as previously described to correspond to a $95 \%$ probability of SARS-CoV-2 neutralization in neutralization tests $[25,26]$. Neutralizing antibody levels were measured on the day of hospitalization in all patients, 7-9 days after hospitalization in patients who did not receive casirivimab/imdevimab therapy, 3-5 days after hospitalization in patients who received casirivimab/imdevimab therapy, and on the day of discharge for all patients.

\section{Comparison of clinical status}

Clinical status of SOTRs with COVID-19 who received and did not receive casirivimab/imdevimab therapy was compared using medical records. Clinical status included the duration until antifebrile; hospitalization duration; body temperature at hospitalization; prognosis; requirement of mechanical ventilation; requirement of oxygen $\left(\mathrm{O}_{2}\right) \geq 5 \mathrm{~L}$ (which is the threshold for applying high flow nasal cannula [27]); level of white blood cells, lymphocytes, hemoglobin, aspartate aminotransferase, alanine aminotransferase, $y$-glutamyl trans-peptidase, creatinine, surfactant protein $D$, Krebs von den lungen-6, Ddimer, albumin, interleukin-6, and C-reactive protein; body mass index; and presence of cardiovascular disease and diabetes mellitus.

\section{Data analysis}

Analysis of data was conducted with Graph Pad PRISM 9 (GraphPad Software, La Jolla, CA, USA) with $t$ tests, Kaplan-Meier analysis, hazard ratio (HR), and 95\% confidence interval (Cl) calculation, which was used to compare the clinical status, neutralizing antibody levels, the duration until antifebrile, and hospitalization duration. SPSS 28 (IBM, Armonk, NY, USA) was used for multiple variables Cox proportional hazards analysis, which was used to compare the duration until antifebrile in SOTRs with or without casirivimab/imdevimab therapy. $P<0.05$ was considered significant.

\section{Results}

Casirivimab/imdevimab correlates with less duration until antifebrile and of hospitalization and nonrequirement of $\mathrm{O}_{2} \geq 5 \mathrm{~L}$ in SOTRs with COVID-19 
Among 41 SOTRs with mild-to-moderate COVID-19 enrolled in this study, 23 patients did not receive casirivimab/imdevimab and the remaining 18 patients received casirivimab/imdevimab. The baseline patient characteristics are summarized in Table $1 \mathrm{~A}$ and $\mathrm{B}$. Casirivimab/imdevimab treatment significantly correlated with less duration until antifebrile (mean days without vs. with casirivimab/imdevimab: 6 vs. 2; $P=0.0002, \mathrm{HR}=0.3333,95 \% \mathrm{Cl}=0.1763-0.6301$ ) and of hospitalization (mean days without vs. with casirivimab/imdevimab: 15 vs. $8 ; P<0.0001, \mathrm{HR}=0.5333,95 \% \mathrm{Cl}=0.2878-0.9883$; Fig. $1 \mathrm{a}$ and $\mathrm{b}$ ) in SOTRs with mild-to-moderate COVID-19.

Furthermore, patients who received casirivimab/imdevimab therapy did not require $\mathrm{O}_{2} \geq 5 \mathrm{~L}$ as compared to those who did not receive this therapy $(P=0.0377, \mathrm{HR}=0.1502,95 \% \mathrm{Cl}=0.02511-0.8980)$.

Casirivimab/imdevimab treatment was significantly associated with the duration until antifebrile after adjusting for sex and age $(P=0.013, \mathrm{HR}=0.412,95 \% \mathrm{Cl}=0.205-0.826$; Table 2$)$. In addition, there were no casualties and mechanical ventilation was not required in patients who received casirivimab/imdevimab therapy, whereas in patients who did not receive casirivimab/imdevimab therapy, one lost life and two required mechanical ventilation.

\section{Casirivimab/imdevimab enhances neutralizing antibody levels in SOTRs with COVID-19}

Neutralizing antibody levels in SOTRs with COVID-19 on each day are presented in Fig. 2a and b. Neutralizing antibody levels was not significantly different between patients without and with casirivimab/imdevimab therapy on the day of hospitalization (mean antibody level without vs. with casirivimab/imdevimab: 7.439 vs. $20.37 ; P=0.1055,95 \% \mathrm{Cl}=-2.854-28.72$; Fig. 3a). Thirteen patients out of 18 who received casirivimab/imdevimab therapy vaccinated within past 8 weeks with BNT162b2 or mRNA-1273 stayed lower neutralizing antibody levels (mean antibody level without vs. with casirivimab/imdevimab: 7.439 vs. $24.22, P=0.072,95 \% \mathrm{Cl}=-1.582-35.13$; Table 1) $[10,11,28]$. Furthermore, casirivimab/imdevimab therapy led to increased neutralizing antibody levels at $3-5$ days after hospitalization in patients with casirivimab/imdevimab therapy compared with the levels at 7-9 days after hospitalization in those without casirivimab/imdevimab therapy (mean antibody level without vs. with casirivimab/imdevimab: 414.9 vs. 40,$000 ; P<0.0001,95 \% \mathrm{Cl}=38,784-40,386$; Fig. 3b). Moreover, neutralizing antibody levels was significantly higher in patients with casirivimab/imdevimab therapy than in those without the therapy at the time of discharge (mean antibody level without vs. with casirivimab/imdevimab: 2,951 vs. 40,$000 ; P<0.0001,95 \% \mathrm{Cl}=34,982-39,116$; Fig. 3c). 
Table 1

a Patient characteristics

\begin{tabular}{|c|c|c|c|c|}
\hline Characteristics & $\begin{array}{l}\text { Without mABs }(n= \\
\text { 23) }\end{array}$ & $\begin{array}{l}\text { With } \mathrm{mABs}(\mathrm{n}= \\
\text { 18) }\end{array}$ & $95 \% \mathrm{Cl}$ & $\begin{array}{l}P \\
\text { value }\end{array}$ \\
\hline Sex male/female & $19 / 4$ & $13 / 5$ & & \\
\hline \multirow[t]{2}{*}{ Vaccinated } & 0 & 13 & & \\
\hline & median (range) & median (range) & & \\
\hline Age & $48.04(20-80)$ & $53.72(26-73)$ & $\begin{array}{l}-3.102- \\
14.46\end{array}$ & 0.199 \\
\hline Body temperature (celsius) & $37.67(36.4-39)$ & $38.14(35.8-40.2)$ & $\begin{array}{l}-0.097- \\
1.038\end{array}$ & 0.105 \\
\hline Body mass index (kg/m2) & $\begin{array}{l}25.39(16.11- \\
47.92)\end{array}$ & $\begin{array}{l}23.54(17.56- \\
33.63)\end{array}$ & $\begin{array}{l}-1.935- \\
5.639\end{array}$ & 0.329 \\
\hline Cardiovascular disease (\%) & 39.13 & 38.89 & $\begin{array}{l}-0.316- \\
0.321\end{array}$ & 0.988 \\
\hline Diabetes mellitus (\%) & 26.09 & 38.89 & $\begin{array}{l}-0.429- \\
0.149\end{array}$ & 0.395 \\
\hline White blood cell $(\mu \mathrm{L})$ & $6302(2350-11570)$ & $\begin{array}{l}5923(3510- \\
9880)\end{array}$ & $\begin{array}{l}-1622- \\
864.4\end{array}$ & 0.541 \\
\hline Lymphocyte cell $(\mu \mathrm{L})$ & $786.2(230-2300)$ & $795.3(270-1908)$ & $\begin{array}{l}-290.7- \\
272.4\end{array}$ & 0.948 \\
\hline Hemoglobin $(\mathrm{g} / \mathrm{dL})$ & $13.47(11-16.2)$ & $13.52(10.6-16)$ & $\begin{array}{l}-0.987- \\
0.901\end{array}$ & 0.928 \\
\hline $\begin{array}{l}\text { Aspartate aminotransferase } \\
(\mathrm{U} / \mathrm{L})\end{array}$ & $30.3(12-148)$ & $23.78(10-58)$ & $\begin{array}{l}-8.253- \\
21.31\end{array}$ & 0.377 \\
\hline $\begin{array}{l}\text { Alanine aminotransferase } \\
(\mathrm{U} / \mathrm{L})\end{array}$ & $27.61(9-108)$ & $14.5(7-43)$ & $\begin{array}{l}0.559- \\
6.205\end{array}$ & 0.041 \\
\hline $\begin{array}{l}\text { y-glutamyl trans-peptidase } \\
(\mathrm{U} / \mathrm{L})\end{array}$ & $77.57(16-628)$ & $53.72(12-207)$ & $\begin{array}{l}-41.26- \\
88.94\end{array}$ & 0.463 \\
\hline Creatinine (mg/dL) & $1.793(0.72-4.44)$ & $1.658(0.74-2.57)$ & $\begin{array}{l}-0.357- \\
0.243\end{array}$ & 0.581 \\
\hline Surfactant protein $\mathrm{D}(\mathrm{ng} / \mathrm{mL})$ & $28.45(17.2-56.5)$ & $21.36(15-84.7)$ & $\begin{array}{l}-5.363- \\
19.55\end{array}$ & 0.256 \\
\hline $\begin{array}{l}\text { Krebs von den lungen-6 } \\
(\mathrm{U} / \mathrm{mL})\end{array}$ & $206.3(113-440)$ & $220.8(115-479)$ & $\begin{array}{l}-76.04- \\
47.18\end{array}$ & 0.638 \\
\hline D-dimer $(\mu \mathrm{g} / \mathrm{mL})$ & $0.687(0.5-2.6)$ & $1.117(0.5-4.3)$ & $\begin{array}{l}-0.916- \\
0.056\end{array}$ & 0.081 \\
\hline
\end{tabular}

$m A B s$, monoclonal antibodies; $95 \% \mathrm{Cl}, 95 \%$ confidence interval 


\begin{tabular}{|c|c|c|c|c|}
\hline Characteristics & $\begin{array}{l}\text { Without mABs }(n= \\
\text { 23) }\end{array}$ & $\begin{array}{l}\text { With mABs }(n= \\
\text { 18) }\end{array}$ & $95 \% \mathrm{Cl}$ & $\begin{array}{l}P \\
\text { value }\end{array}$ \\
\hline Albumin (g/dL) & $4.1(3.1-4.8)$ & $3.9(2.8-4.6)$ & $\begin{array}{l}-0.105- \\
0.505\end{array}$ & 0.193 \\
\hline Interleukin-6 (pg/dL) & $31.8(1.4-289)$ & $20.79(2.8-61.7)$ & $\begin{array}{l}-20.84- \\
42.86\end{array}$ & 0.489 \\
\hline C-reactive protein (mg/dL) & $4.11(0.1-36.32)$ & $3.67(0.33-10.24)$ & $\begin{array}{l}-3.542- \\
4.501\end{array}$ & 0.811 \\
\hline Neutralizing antibody $(\mathrm{AU} / \mathrm{mL})$ & $7.439(6.8-15.7)$ & $10.38(6.8-24.7)$ & $\begin{array}{l}-0.743- \\
6.625\end{array}$ & 0.113 \\
\hline Vaccinated (AU/mL) & & $24.22(6.8-164.3)$ & $\begin{array}{l}-1.582- \\
35.13\end{array}$ & 0.072 \\
\hline
\end{tabular}

Table 1

b The organ of transplant

\begin{tabular}{|lll|}
\hline Transplant organ & Without mABs $(\mathbf{n = 2 3 )}$ & With mABs $(\mathrm{n}=18)$ \\
\hline Kidney & 22 & 14 \\
Liver & 0 & 3 \\
\hline Bone marrow & 1 & 1 \\
\hline mABs, monoclonal antibodies & \\
\hline
\end{tabular}

Table 2

Multiple variables Cox proportional hazards analysis for antifebrile in SOTRs

\begin{tabular}{|lllllll|}
\hline & \multicolumn{3}{c}{ Univariate analysis } & \multicolumn{2}{c|}{ Multivariate analysis } \\
\hline Covariate & HR & $95 \% \mathrm{Cl}$ & $P$ value & HR & $95 \% \mathrm{Cl}$ & $P$ value \\
\hline Sex & 1.137 & $0.499-2.595$ & 0.760 & & & \\
\hline Age & 0.994 & $0.971-1.016$ & 0.579 & & & \\
\hline Casirivimab/imdevimab & 0.412 & $0.205-0.826$ & 0.013 & 0.412 & $0.205-0.826$ & 0.013 \\
\hline \multicolumn{5}{|l|}{ SOTRs, solid organ transplant recipients; $H R$, hazards ratio; $95 \% \mathrm{Cl}, 95 \%$ confidence interval } \\
\hline
\end{tabular}

\section{Discussion}


Accumulating evidence has demonstrated that neutralizing antibody, including casirivimab/imdevimab, bamlanivimab/etsevimab, and sotrovimab, are associated with lesser hospitalization duration and death rate for high-risk patients and SOTRs with mild-to-moderate COVID-19 [5, 14-22].

In this study, we assessed the efficacy of casirivimab/imdevimab therapy in SOTRs with mild-tomoderate COVID-19. Specifically, we demonstrated that casirivimab/imdevimab therapy correlated with less duration until antifebrile and of hospitalization and non-requirement of $\mathrm{O}_{2} \geq 5 \mathrm{~L}$ in SOTRs with mildto-moderate COVID-19 (Figs. 1 and 2). In addition, casirivimab/imdevimab therapy increased neutralizing antibody levels in SOTRs with COVID-19 (Fig. 3b and C), suggesting that it is appropriate to apply casirivimab/imdevimab therapy for such patients. These results should be related to the observation that casirivimab/imdevimab therapy reduced duration of hospitalization, acute respiratory failure, and death rate in SOTRs with COVID-19, as previously described [5, 17-22]. Furthermore, neutralizing antibody levels in SOTRs with COVID-19 who received casirivimab/imdevimab therapy (Fig. 3a) including 13 vaccinated patients (Table 1) were not high enough on the day of hospitalization compared with those in patients who did not receive casirivimab/imdevimab therapy to prevent breakthrough SARS-CoV-2 infection [29]. In addition to immunosuppression, a poor neutralizing antibody levels similar to that without vaccination or without casirivimab/imdevimab therapy must be one of the reasons why the 13 patients acquired a breakthrough SARS-CoV-2 infection despite vaccination. A previous study reported that IgG and the neutralizing antibody levels against COVID-19 increased until 4-20 days after COVID-19 onset [30,31]; however, our results demonstrated that SOTRs with COVID-19 who did not receive casirivimab/imdevimab therapy did not have enough neutralizing antibody levels even 7-9 days after hospitalization (Fig. 3b) and at discharge (Fig. 3c) to prevent the progression of COVID-19 and the subsequent SARS-CoV-2 infection. These results suggested that compared with those who receive casirivimab/imdevimab therapy, SOTRs with COVID-19 who do not receive casirivimab/imdevimab therapy may need long duration until antifebrile and of hospitalization, may have severe COVID-19 with $\mathrm{O}_{2}$ requirement $\geq 5 \mathrm{~L}$, and may reacquire COVID-19 even right after discharge.

Based on these results, casirivimab/imdevimab therapy is more effective against mild-to-moderate COVID-19 in SOTRs than therapy without casirivimab/imdevimab. As casirivimab/imdevimab enhanced neutralizing antibody levels in SOTRs with COVID-19, it could contribute to less duration until antifebrile and of hospitalization and non-requirement of $\mathrm{O}_{2} \geq 5 \mathrm{~L}$ in SOTRs with COVID-19. Therefore, casirivimab/imdevimab therapy is efficient in preventing the progression of mild-to-moderate COVID-19 in SOTRs.

This study has some limitations. Firstly, it was retrospectively performed at a single hospital with a small number of SOTRs, which may have influenced the results, and did not conduct a randomized controlled study to evaluate the results. Secondly, it was performed only in Tokyo, and the situation may be different in other areas globally. Finally, implementing this neutralizing mAbs is costly. Therefore, less costly treatment options need to be developed.

\section{Conclusions}


Our results demonstrated that casirivimab/imdevimab therapy involving neutralizing mAbs is effective against mild-to-moderate COVID-19 in SOTRs compared with treatment without casirivimab/imdevimab. Casirivimab/imdevimab enhanced neutralizing antibody levels against COVID-19 in SOTRs in whom the level tended to stay lower despite vaccination with BNT162b2 or mRNA-1273. Therefore, casirivimab/imdevimab therapy could contribute to preventing the progression of mild-to-moderate COVID-19 in SOTRs.

\section{Abbreviations}

COVID-19, coronavirus disease 2019; mAbs, monoclonal antibodies; PCR, polymerase chain reaction; SARS-CoV-2, severe acute respiratory syndrome coronavirus 2; SOTRs, solid organ transplant recipients.

\section{Declarations}

\section{Ethics approval and consent to participate}

The Institutional Review Board of Tokyo Women's Medical University Hospital approved this study (date of approval: January 5, 2022; approval number: 2021-0175). Informed consent was obtained from all the patients in this study. All methods were carried out in accordance with relevant guidelines and regulations.

\section{Consent for publication}

Not applicable.

\section{Availability of data and materials}

Data that supports the conclusions of this study is demonstrated in this manuscript. The clinical detail of data is available from all corresponding author and author. However, data has not been made available publicly.

\section{Competing interests}

The authors declare that they have no competing interests.

\section{Funding}

Not received

\section{Author's contributions}

$\mathrm{KA}$ and $\mathrm{HS}$ conceptualized and designed the study. KA, FE, TM, KU, TK, HI, HE, and JT performed data acquisition. $\mathrm{KA}, \mathrm{YH}, \mathrm{HS}$, and $\mathrm{KS}$ analyzed the data. $\mathrm{KA}, \mathrm{ET}$, and $\mathrm{MK}$ wrote the manuscript $\mathrm{KA}, \mathrm{KK}, \mathrm{TM}, \mathrm{ET}$, 
and $\mathrm{MK}$ revised and edited the manuscript. MK, KT, and TK supervised the study. All authors have read and approved the manuscript.

\section{Acknowledgements}

The authors would like to thank Hitomi Miura for her assistance.

\section{References}

1. Hick JL, Biddinger PD. Novel coronavirus and old lessons - preparing the health system for the pandemic. N Engl J Med. 2020;382:e55.

2. Coronavirus disease (COVID-19) Weekly Epidemiological Update and Weekly Operational Update. World Health Organization. (https://www.who.int/emergencies/diseases/novel-coronavirus-2019/situationreports)

3. Guan WJ, Liang WH, Zhao Y, Liang HR, Chen ZS, Li YM, Liu XQ, Chen RC, Tang CL, Wang T, et al. Comorbidity and its impact on 1590 patients with COVID-19 in China: a nationwide analysis. Eur Respir J. 2020;55:2000547.

4. Lerner AH, Klein E, Farmakiotis D. Mortality in organ transplant recipients with Covid-19 compared to non-transplant or waitlisted patients: a meta-analysis. Am J Transplant. 2021;35:100628.

5. Klein EJ, Hardesty A, Vieira K, Farmakiotis D. Use of anti-spike monoclonal antibodies in kidney transplant recipients with COVID-19: Efficacy, ethnic and racial disparities. Am J Transplant. 2021 doi: 10.1111/ajt.16843. Online ahead of print.

6. Beigel JH, Tomashek KM, Dodd LE, Mehta AK, Zingman BS, Kalil AC, Hohmann E, Chu HY, Luetkemeyer A, Kline S, et al. Remdesivir for the treatment of COVID-19 - Final report. N Engl J Med. 2020;383:1813-26.

7. Horby P, Lim WS, Emberson JR, Mafham M, Bell JL, Linsell L, Staplin N, Brightling C, Ustianowski A, Elmahi E, et al. Dexamethasone in hospitalized patients with COVID-19. N Engl J Med. 2021;384:693-704.

8. Gordon AC, Mouncey PR, Al-Beidh F, Rowan KM, Nichol AD, Arabi YM, Annane D, Beane A, van BentumPuijk W, Berry LR, et al. Interleukin-6 receptor antagonists in critically ill patients with COVID-19. N Engl J Med. 2021;384:1491-1502.

9. Kalil AC, Patterson TF, Mehta AK, Tomashek KM, Wolfe CR, Ghazaryan V, Marconi VC, Ruiz-Palacios GM, Hsieh L, Kline S, et al. Baricitinib plus remdesivir for hospitalized adults with COVID-19. N Engl J Med. 2021;384:795-807.

10. Polack FP, Thomas SJ, Kitchin N, Absalon J, Gurtman A, Lockhart S, Perez JL, Pérez Marc G, Moreira ED, Zerbini C, et al. Safety and efficacy of the BNT162b2 mRNA COVID-19 vaccine. N Engl J Med. 2020;383:2603-15. 
11. Baden LR, El Sahly HM, Essink B, Kotloff K, Frey S, Novak R, Diemert D, Spector SA, Rouphael N, Creech CB, et al. Efficacy and safety of the mRNA-1273 SARS-CoV-2 vaccine. N Engl J Med. 2021;384:403-16.

12. Caillard S, Chavarot N, Francois H, Matignon M, Greze C, Kamar N, Gatault P, Thaunat O, Legris T, Frimat L, et al. Is COVID-19 infection more severe in kidney transplant recipients? Am J Transplant. 2021;21:1295-1303.

13. Boyarsky BJ, Werbel WA, Avery RK, Tobian AAR, Massie AB, Segev DL, Garonzik-Wang JM. Antibody response to 2-dose SARS-CoV-2 mRNA vaccine series in solid organ transplant recipients. JAMA. 2021;325:2204-6.

14. Weinreich DM, Sivapalasingam S, Norton T, Ali S, Gao H, Bhore R, Xiao J, Hooper AT, Hamilton JD, Musser BJ, et al. REGEN-COV antibody combination and outcomes in outpatients with COVID-19. N Engl J Med. 2021. doi: 10.1056/NEJMoa2108163. Online ahead of print.

15. Dougan M, Nirula A, Azizad M, Mocherla B, Gottlieb RL, Chen P, Hebert C, Perry R, Boscia J, Heller B, et al. Bamlanivimab plus etesevimab in mild or moderate COVID-19. N Engl J Med. 2021;385:1382-92.

16. Gupta A, Gonzalez-Rojas Y, Juarez E, Crespo Casal M, Moya J, Falci DR, Sarkis E, Solis J, Zheng H, Scott N, et al. Early treatment for COVID-19 with SARS-CoV-2 neutralizing antibody sotrovimab. N Engl J Med. 2021;385:1941-50.

17. Del Bello A, Marion O, Vellas C, Faguer S, Izopet J, Kamar N. Anti-SARS-CoV-2 monoclonal antibodies in solid-organ transplant patients. Transplantation. 2021;105:e146-7.

18. Dhand A, Lobo SA, Wolfe K, Feola N, Lee L, Nog R, Chen D, Glicklich D, Diflo T, Nabors C. Casirivimabimdevimab for treatment of COVID-19 in solid organ transplant recipients: an early experience. Transplantation. 2021;105:e68-9.

19. Hasan L, Hardgrave H, Osborn T, Burdine L, Giorgakis E, Rico J. Monoclonal antibodies for Covid-19 in patients with solid organ transplant recipients. Am J Transplant. 2021;21. https://atcmeeting abstracts.com/abstract/monoclonal-antibodies-for-covid-19-in- patients-with-solid-organ-transplantrecipients/.

20. Jan MY, El-Sayegh S, Yaqub MS, Yaqub D. P Mishler, O Adebiyi, M. D. Anderson, T Taber, A Sharfuddin. Bamlanivimab for COVID- 19 in kidney transplant patients. Am J Transplant. 2021;21. https://atcmeeting abstracts.com/abstr act/bamlanivimab-for-covid-19-in-kidney-transplant-patients/.

21. Monoclonal antibody therapy for COVID-19 in solid organ transplant recipients.

Yetmar ZA, Beam E, O'Horo JC, Ganesh R, Bierle DM, Brumble L, Seville MT, Razonable RR. Open Forum Infect Dis. 2021;8:ofab255. doi: 10.1093/ofid/ofab255. 
22. Kutzler HL, Kuzaro HA, Serrano OK, Feingold A, Morgan G, Cheema F. Initial experience of bamlanivimab monotherapy use in solid organ transplant recipients. Transpl Infect Dis. 2021;e13662. doi:10.1111/tid.13662

23. Food and Drug Administration. COVID-19: developing drugs and biological

products for treatment or prevention. (https://www.fda.gov)

24. Arimura K, Tagaya E, Kikuchi K, Mitsuda T, Yasuda G, Azuma H, Konda N, Ikarashi Y, Chiba Y, Kusama $\mathrm{K}$, Sakama R, et al. Polymerase-chain reaction testing to prevent hospital-acquired severe acute respiratory syndrome coronavirus 2 infection in Shinjuku, an epicenter in Tokyo: The Tokyo Women's Medical University model.

Respir Investig. 2021;59:356-9.

25. Ebinger JE, Fert-Bober J, Printsev I, Wu M, Sun N, Prostko JC, Frias EC, Stewart JL, Van Eyk JE, Braun $\mathrm{JG}$, et al. Antibody responses to the BNT162b2 mRNA vaccine in individuals previously infected with SARS-CoV-2. Nat Med. 2021;27:981-4.

26. Redjoul R, Le Bouter A, Beckerich F, Fourati S, Maury S. Antibody response after second BNT162b2 dose in allogeneic HSCT recipients. Lancet. 2021.398(10297):298-9.

27. Perkins GD, Couper K, Connolly B, Baillie JK, Bradley JM, Dark P, De Soyza A, Gorman E, Gray A, Hamilton L, et al. RECOVERY- Respiratory Support: Respiratory Strategies for patients with suspected or proven COVID-19 respiratory failure; Continuous Positive Airway Pressure, High-flow Nasal Oxygen, and standard care: A structured summary of a study protocol for a randomised controlled trial.

Trials. 2020;21:687.

28. Thompson MG, Burgess JL, Naleway AL, Tyner H, Yoon SK, Meece J, Olsho LEW, Caban-Martinez AJ, Fowlkes AL, Lutrick K, et al. Prevention and attenuation of COVID-19 with the BNT162b2 and mRNA-1273 vaccines. N Engl J Med. 2021;385:320-9.

29. Bergwerk M, Gonen T, Lustig Y, Amit S, Lipsitch M, Cohen C, Mandelboim M, Levin EG, Rubin C, Indenbaum V, et al. COVID-19 breakthrough infections in vaccinated health care workers. N Engl J Med. 2021;385:1474-84.

30. Nakano Y, Kurano M, Morita Y, Shimura T, Yokoyama R, Qian C, Xia F, He F, Kishi Y, Okada J, et al. Time course of the sensitivity and specificity of anti-SARS-CoV-2 IgM and IgG antibodies for symptomatic COVID-19 in Japan. Sci Rep. 2021;11:2776.

31. Seydoux E, Homad LJ, MacCamy AJ, Parks KR, Hurlburt NK, Jennewein MF, Akins NR, Stuart AB, Wan $\mathrm{YH}$, Feng J, et al. Analysis of a SARS-CoV-2-infected individual reveals development of potent neutralizing antibodies with limited somatic mutation. Immunity. 2020;53:98-105. 
Figures
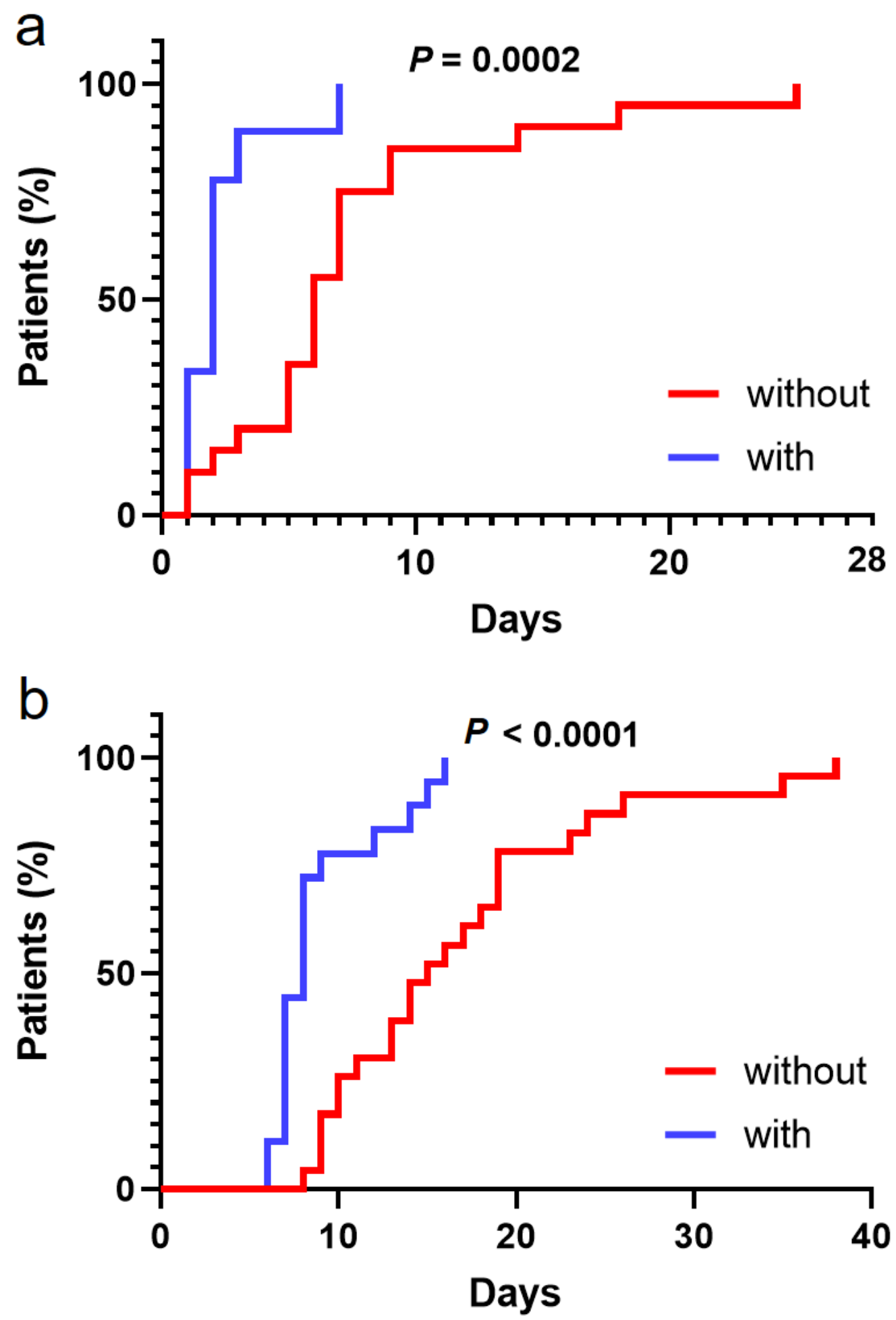

Figure 1

The duration until antifebrile and of hospitalization since hospitalization in SOTRs with COVID-19 who received and did not receive casirivimab/imdevimab therapy. a, Casirivimab/imdevimab therapy correlated with less duration until antifebrile in SOTRs with mild-to-moderate COVID-19 than that in 
SOTRs who did not receive casirivimab/imdevimab $(P=0.0002, \mathrm{HR}=0.3333,95 \% \mathrm{Cl}=0.1763-0.6301)$.

b, Casirivimab/imdevimab therapy correlated with early discharge in SOTRs with mild-to-moderate COVID-19 compared with that in SOTRs who did not receive casirivimab/imdevimab $(P<0.0001, \mathrm{HR}=$ $0.5333,95 \% \mathrm{Cl}=0.2878-0.9883)$.

$\mathrm{O}_{2}$, oxygen; COVID-19, coronavirus disease 2019; SOTRs, solid organ transplant recipients; HR, hazard ratio; $95 \% \mathrm{Cl}$, 95\% confidence interval; without, without casirivimab/imdevimab; with, with casirivimab/imdevimab. 

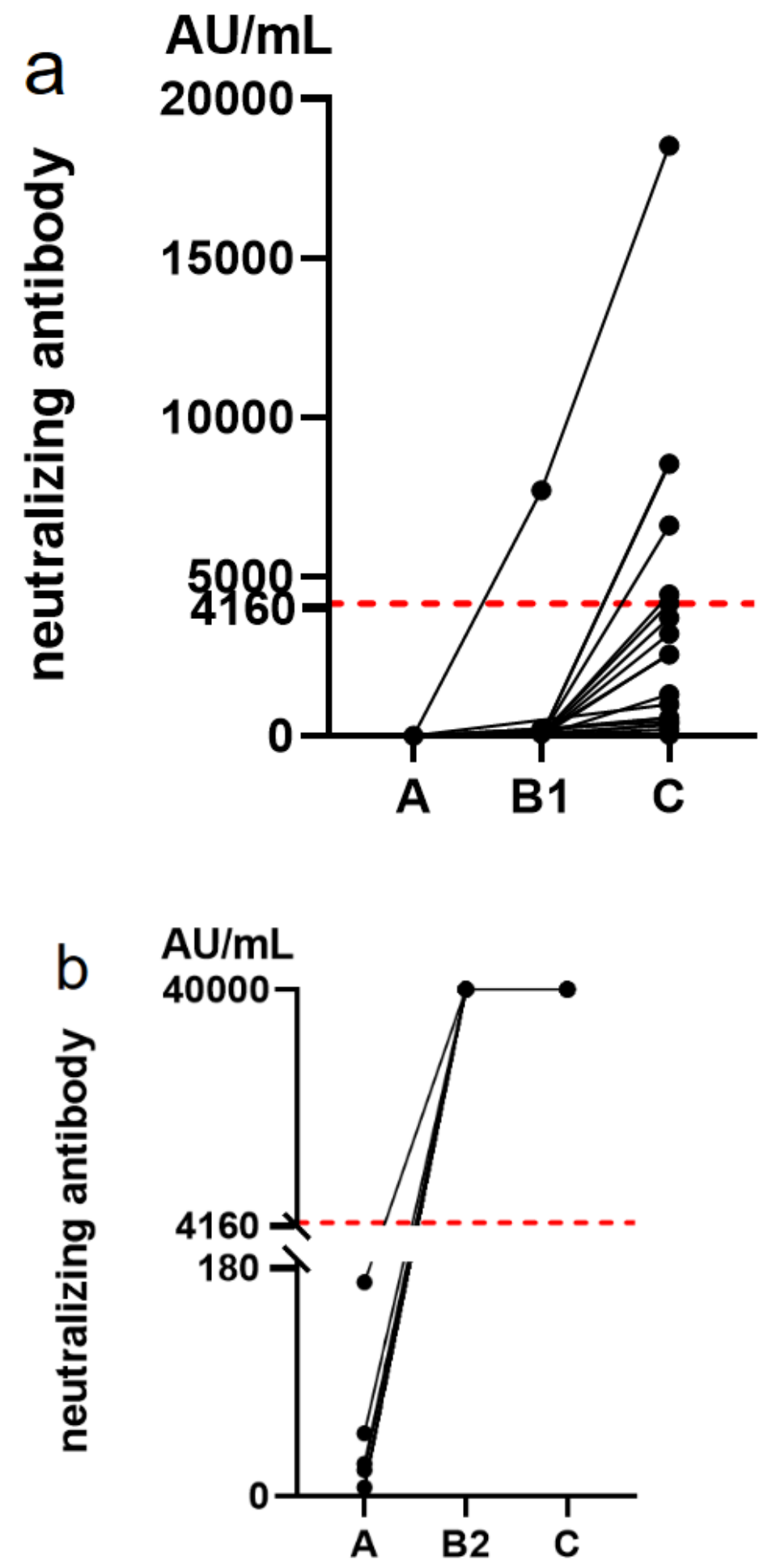

Figure 2

Neutralizing antibody levels in SOTRs with mild-to-moderate COVID-19. Neutralizing antibody levels in patients a, who did not receive casirivimab/imdevimab therapy and $b$, who received casirivimab/imdevimab therapy. 
A, day of hospitalization; B1, 7-9 days after hospitalization; B2, 3-5 days after hospitalization; C, day of discharge.

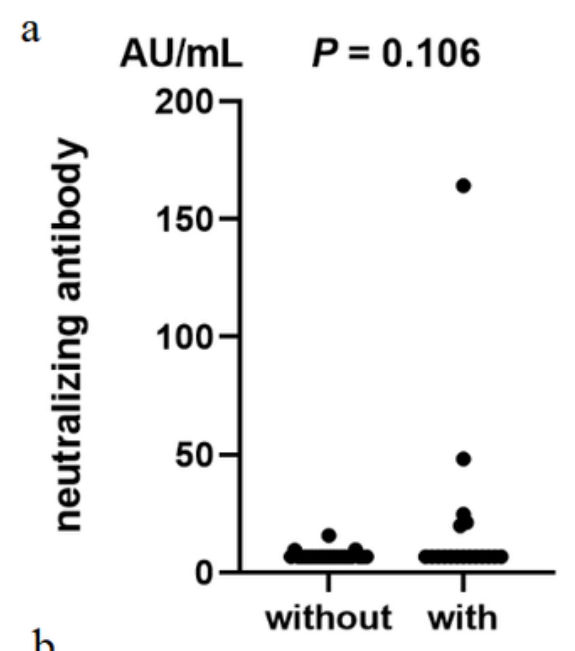

b

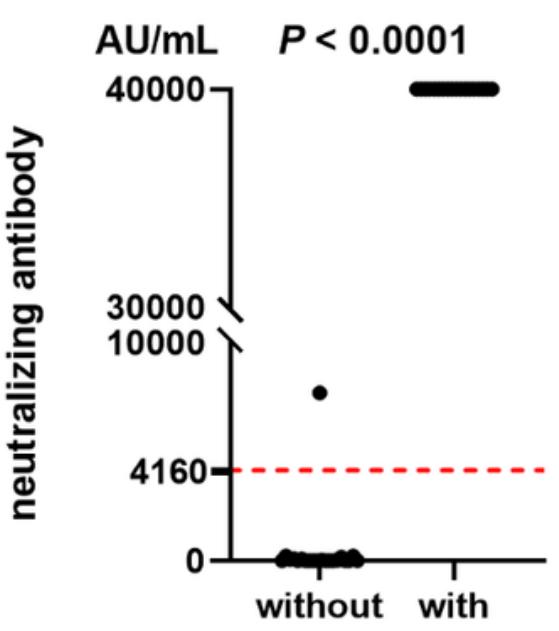

c

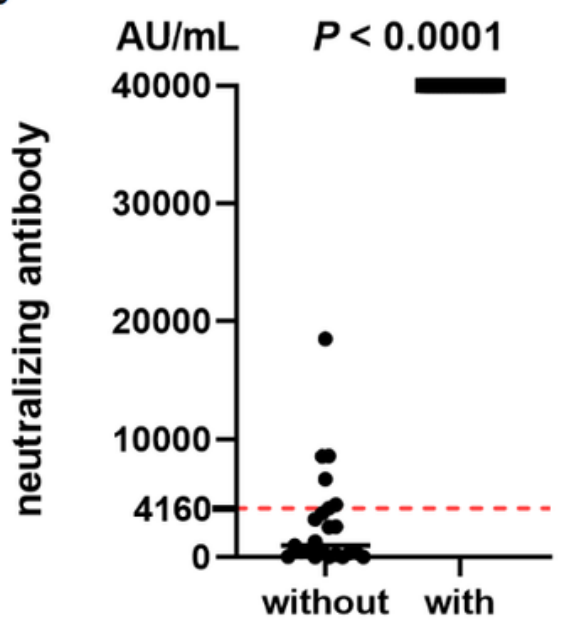

Figure 3

Neutralizing antibody levels in SOTRs with mild-to-moderate COVID-19 who received and did not receive casirivimab/imdevimab therapy. a, No significant difference was observed between the two groups of 
patients at the day of hospitalization (mean antibody level without vs. with casirivimab/imdevimab 7.439 vs. $20.37 ; P=0.1055,95 \% \mathrm{Cl}=-2.854-28.72$ ) including 13 SOTRs vaccinated in past 8 weeks with BNT162b2 or mRNA-1273 who received casirivimab/imdevimab. b, Neutralizing antibody levels were enhanced after casirivimab/imdevimab therapy at 3-5 days after hospitalization compared with those at 7-9 days after hospitalization in patients who did not receive casirivimab/imdevimab therapy (mean antibody level without vs. with casirivimab/imdevimab: 414.9 vs. $40000, P<0.0001,95 \% \mathrm{Cl}=38784-$ 40386). c, At discharge, neutralizing antibody levels was significantly higher in patients who received casirivimab/imdevimab therapy than in those who did not receive the therapy (mean antibody level without vs. with casirivimab/imdevimab: 2951 vs. $40000, P<0.0001,95 \% \mathrm{Cl}=34982-39116)$.

without, without casirivimab/imdevimab; with, with casirivimab/imdevimab. 